Décadrages Décadrages

cinéma, à travers champs Cinéma, à travers champs

29-30 | 2015

René Vautier

\title{
La Geste de l'eau, scénario de René Vautier
}

\section{Olivier Hadouchi}

\section{(C) OpenEdition}

\section{Journals}

Édition électronique

URL : https://journals.openedition.org/decadrages/808

DOI : $10.4000 /$ decadrages. 808

ISSN : 2297-5977

\section{Éditeur}

Association Décadrages

\section{Édition imprimée}

Date de publication : 1 avril 2015

Pagination : 168-176

ISBN : 9782970096306

ISSN : 2235-7823

Référence électronique

Olivier Hadouchi, «La Geste de l'eau, scénario de René Vautier », Décadrages [En ligne], 29-30 | 2015, mis en ligne le 01 mars 2017, consulté le 26 mars 2023. URL : http://journals.openedition.org/ decadrages/808; DOI : https://doi.org/10.4000/decadrages.808

\section{(c) (i) (2)(2)}

Creative Commons - Attribution - Pas d'Utilisation Commerciale - Partage dans les Mêmes Conditions 4.0 International - CC BY-NC-SA 4.0

https://creativecommons.org/licenses/by-nc-sa/4.0/ 


\section{La Geste de l'eau, scénario de René Vautier}

Ce teXte, PréSEnté comme un scénARIo non tourné de René Vautier, peut surprendre de prime abord: le lecteur a l'impression de se trouver face au scénario d'un film de Jean Rouch plutôt qu'à un projet porté par l'auteur de L'Algérie en flammes. Ne ressemble-t-il pas à une espèce de conte ethnographique ou à la transcription d'un conte traditionnel africain aux allures de fable? Il met en scène deux ethnies africaines, les Dogons et les Peuhls, qui vivaient sur des territoires désormais à cheval sur plusieurs nations, sur des frontières héritées de la colonisation. René Vautier a probablement puisé une partie de son inspiration dans son parcours personnel: durant le tournage d'Afrique 5o, en Côte d'Ivoire et au Mali, il a vécu caché pendant quelque temps, protégé par des Dogons (au nord du Mali), pour échapper à la police coloniale. L'aspect pédagogique a toujours été un élément central du cinéma de René Vautier; rappelons que c'est la Ligue de l'Enseignement qui avait commandité le film Afrique 5o, et cet aspect est développé de manière à la fois très subtile et très dense dans des films tels que Avoir 20 ans dans les Aurès ou La Folle de Toujane. René Vautier est avant tout un pédagogue progressiste, soucieux de susciter une réflexion et une action, en ayant souvent recours au registre du conte afin d'enseigner de façon vivante et imagée (voir Avoir 20 ans dans les Aurès, Le Remords, La Folle de Toujane, Les Ajoncs, et bien sûr Afrique 5o).

La Geste de l'eau apparaît comme une sorte d'allégorie, un conte et une fable dédiés à la solidarité, à l'internationalisme: une invitation à aller au-delà des différences ethniques et nationales, afin d'œuvrer au rassemblement pour le bien de tous. Dans le même registre que ce «plaidoyer pour l'unité africaine», rappelons que René Vautier a participé à L'Aube des damnés d'Ahmed Rachedi, vigoureux pamphlet anticolonialiste, vibrant à l'unité du tiers-monde contre l'impérialisme. De plus, derrière ce scénario inédit, on peut voir aussi un appel à la création d'un nouveau type de rapports entre la France et l'Algérie, qui sort d'une guerre particulièrement violente et meurtrière. 
Signalons que durant les premiers mois de sa parution, Révolution africaine comportait une équipe mixte de journalistes, avec des Algériens et des Français (solidaires de leur combat et de leur aspiration au changement) tels que Gérard Chaliand, Jacques Vergès, Juliette Minces, Elie Kagan, Siné ou Georges Arnaud. Organe central du FLN, l'hebdomadaire accordait une très large importance aux questions internationales, proposant régulièrement des portraits et des entretiens avec des dirigeants de mouvements de libération ou des personnalités politiques comme Nelson Mandela, le président Nasser, Che Guevara, Mao Tse Toung, Ho Chi Minh - mentionnons aussi plusieurs pages en hommage à Frantz Fanon. Il s'intéressait aux arts tels que la sculpture, la littérature ou le cinéma; Guy Hennebelle y écrivait des textes sur le cinéma sous le pseudonyme de Kamel Bensaid (et sous le nom d'Halim Chergui dans le quotidien du parti, El Moudjahid). Révolution africaine a donné la parole aux étudiants de René Vautier, à l'équipe de cinéastes et de techniciens (formés par lui) ayant tourné Peuple en marche (1962-1963) sous sa direction; l'hebdomadaire a également évoqué l'expérience des «ciné-pops» menée par René Vautier en Algérie, et qui consistait à proposer un cinéclub itinérant dans les endroits reculés du pays, en écho à l'expérience des «ciné-mobiles» qui se développaient à Cuba à la même époque, afin de susciter des débats et des prises de conscience, et de relayer des mots d'ordre révolutionnaires.

Au Mali, depuis les bords du Niger, la plaine sèche s'étend vers l'est, mollement ondulée. Des kilomètres et des kilomètres de plaine, verte à la brève saison des pluies, d'or ou de cendre toute l'année; et, tout au bout de la plaine, coupant l'horizontalité, la formidable muraille ocre de Bendiagara, une falaise de plus de roo mètres d'à-pic, de plus de zoo kilomètres de long...

Au pied de cette falaise coule une rivière, toujours alimentée, même aux plus fortes chaleurs, par une cascade jaillissant d'une grotte, au flanc de la roche abrupte.

Là, deux mondes sont en présence: un monde hori- zontal, celui de la plaine; un monde vertical, celui de la falaise. Deux peuples en présence: le peuple peuhl, fils des grands espaces plats où errent les troupeaux; le peuple dogon, fils du rocher, où ne peuvent vivre d'autres animaux domestiques que des chèvres et des chiens, un peuple de jardiniers, d'agriculteurs... Entre ces deux peuples, seul point commun, source de multiples contestations: la rivière...

JADIS, IL Y A DE CELA DES SIÈCLES et des siècles, les Dogons cultivaient la plaine, tout le long de la rivière, 


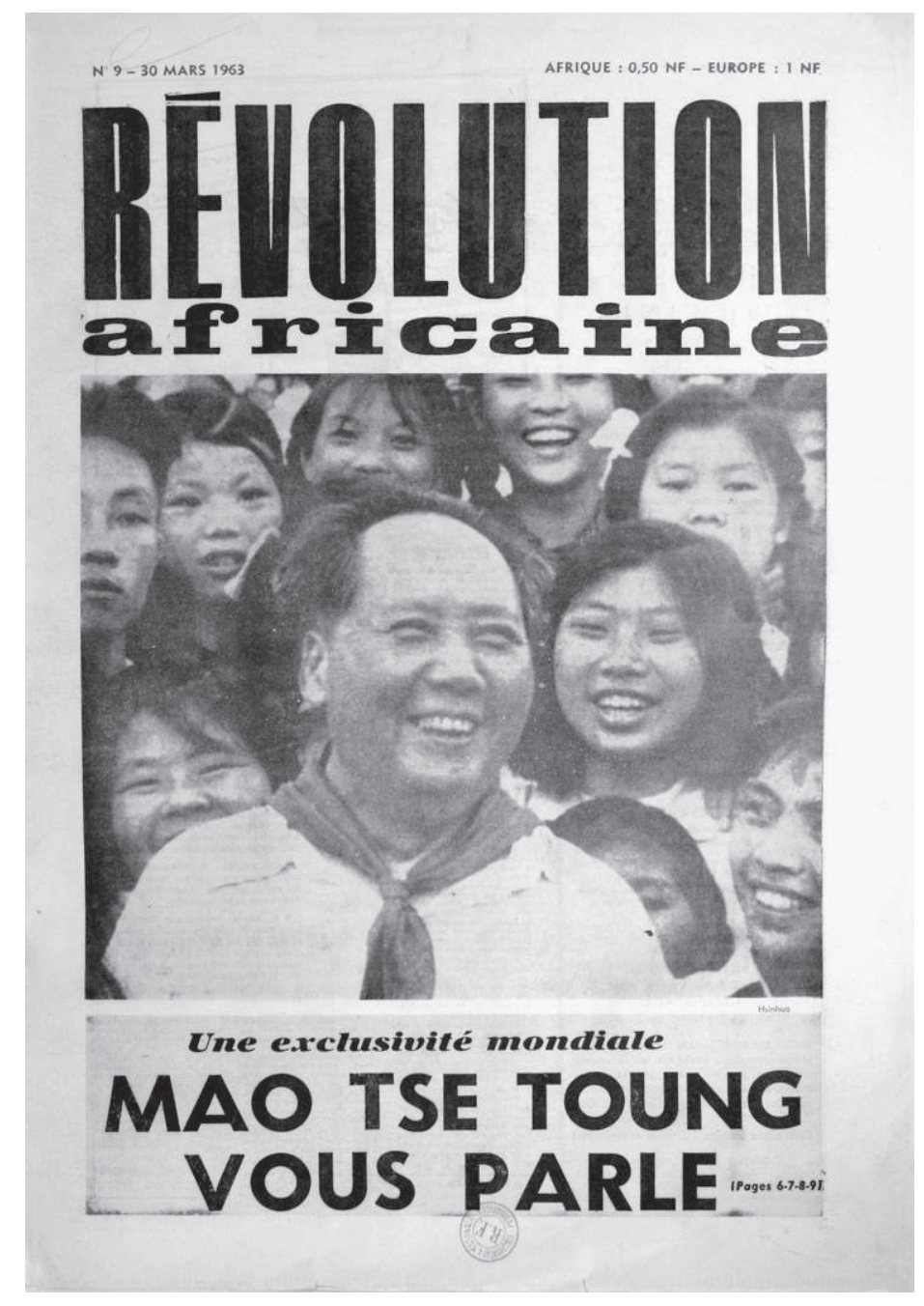

et leurs villages se tenaient au bord de l'eau. Mais les Peuhls, venant de lointains horizons, accoururent en vagues successives, poussant leurs troupeaux vers l'eau, piétinant les cultures, ruinant tout... Les Dogons, les armes à la main, repoussèrent les premières tribus; mais d'autres s'en venaient inlassablement, et les villages dogons succombèrent en ces combats toujours recommencés... Fils du rocher, ils retournèrent au rocher, à la falaise; leurs maisons s'accrochèrent aux rares aspérités du roc, hors de portée 

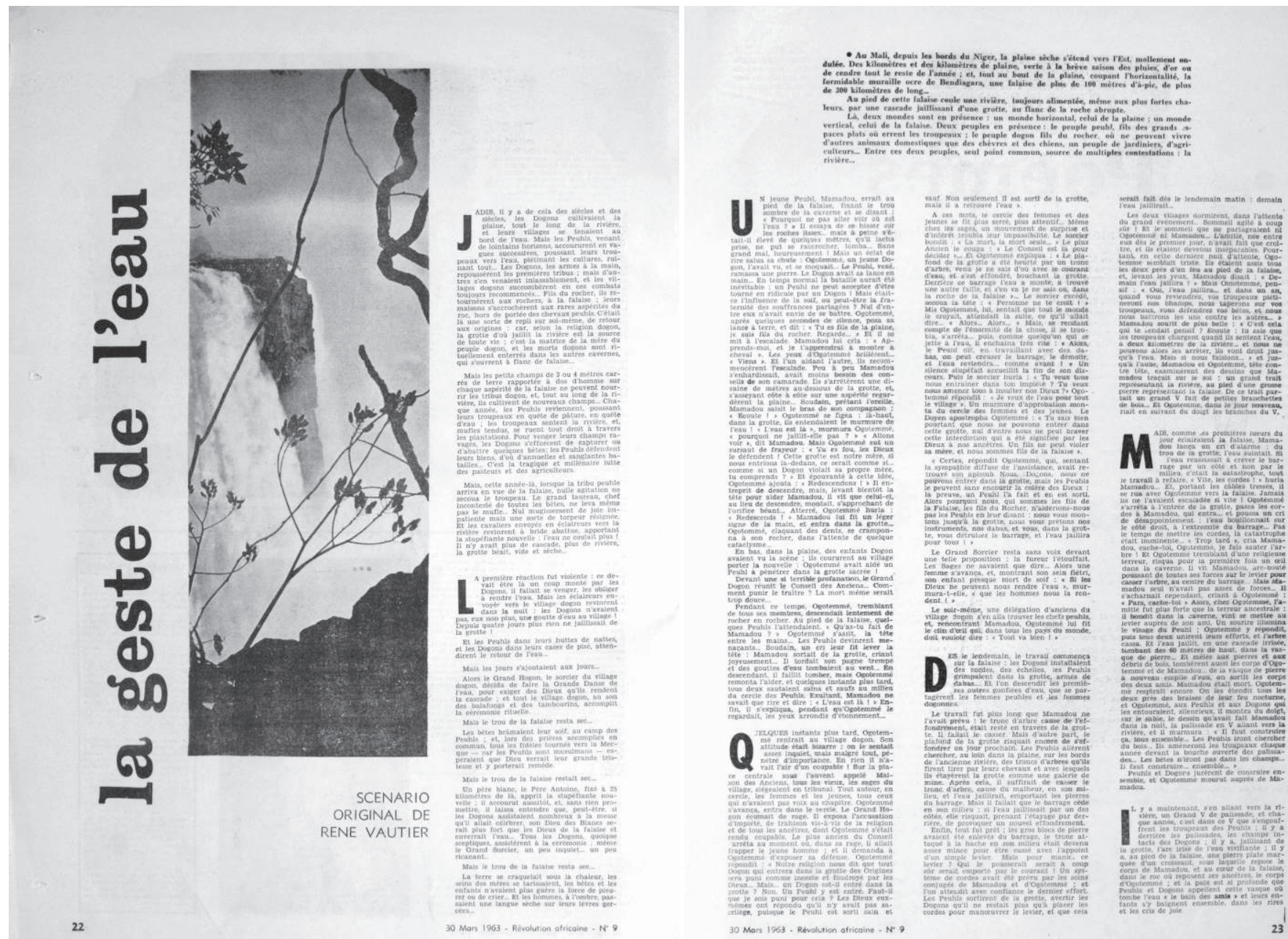

des chevaux peuhls. C'était là une sorte de repli sur soi-même, de retour aux origines: car, selon la religion dogon, la grotte d'où jaillit la rivière est la source de toute vie; c'est la matrice de la mère du peuple dogon, et les morts dogons sont rituellement enter- rés dans les autres cavernes, qui s'ouvrent à flanc de falaise...

Mais les petits champs de 3 ou 4 mètres carrés de terre rapportée à dos d'homme sur chaque aspérité de la falaise ne peuvent nourrir les tribus dogons, et, tout 
au long de la rivière, ils cultivent de nouveaux champs. Chaque année, les Peuhls reviennent, poussant leurs troupeaux en quête de pâture, en quête d'eau; les troupeaux sentent la rivière, et, mufles tendus, se ruent tout droit à travers leurs plantations. Pour venger leurs champs ravagés, les Dogons s'efforcent de capturer ou d'abattre quelques bêtes; les Peuhls défendent leurs biens, d'où d'annuelles et sanglantes batailles... C'est la tragique et millénaire lutte des pasteurs et des agriculteurs.

Mais, cette année-là, lorsque la tribu peuhle arriva en vue de la falaise, nulle agitation ne secoua le troupeau. Le grand taureau, chef incontesté de toutes les bêtes ne leva même pas le mufle... Nul mugissement de joie impatiente mais une sorte de torpeur résignée. Et les cavaliers envoyés en éclaireurs vers la rivière revinrent à bride abattue, apportant la stupéfiante nouvelle: l'eau ne coulait plus! Il n'y avait plus de cascade, plus de rivière, la grotte béait, vide et sèche...

LA PREMIÈRE RÉACTION fut violente; ce devait être là un coup monté par les Dogons, il fallait se venger, les obliger à rendre l'eau. Mais les éclaireurs envoyés au village dogon revinrent dans la nuit: les Dogons n'avaient pas, eux non plus, une goutte d'eau au village! Depuis quatre jours plus rien ne jaillissait de la grotte!

Et les Peuhls dans leurs huttes de nattes, et les Dogons dans leurs cases de pisé, attendirent le retour de l'eau...

Mais les jours s'ajoutaient aux jours...

Alors, le grand Hogon, le sorcier du village dogon, décida de faire la Grande Danse de l'eau, pour exiger des Dieux qu'ils rendent la cascade; et tout le village dogon, au son des balafongs et des tambourins, accomplit la cérémonie rituelle.

Mais le trou de la falaise resta sec...
Les bêtes brâmaient leur soif, au camp des Peuhls, et, lors des prières accomplies en commun, tous les fidèles tournés vers la Mecque - car les Peuhls sont musulmans - espéraient que Dieu verrait leur grande tristesse et y porterait remède.

Mais le trou de la falaise resta sec...

Un père blanc, le Père Antoine, fixé à 25 kilomètres de là, apprit la stupéfiante nouvelle; il accourut aussitôt; et, sans rien promettre, il laissa entendre que, peut-être, si les Dogons assistaient nombreux à la messe qu'il allait célébrer, son Dieu des Blancs serait plus fort que les Dieux de la falaise et enverrait l'eau... Tous les Dogons, quoique sceptiques, assistèrent à la cérémonie; même le Grand Sorcier, un peu inquiet, un peu ricanant...

Mais le trou de la falaise resta sec...

La terre se craquelait sous la chaleur, les seins des mères se tarissaient, les bêtes et les enfants n'avaient plus guère la force de pleurer ou de crier... Et les hommes, à l'ombre, passaient une langue sèche sur leurs lèvres gercées...

Un jeune Peuhl, Mamadou, errait au pied de la falaise, fixant le trou sombre de la caverne et se disant: «Pourquoi ne pas essayer de voir où est l'eau?» Il essaya de se hisser sur les roches lisses... mais à peine s'était-il élevé de quelques mètres, qu’il lâcha prise, ne put se raccrocher, tomba... Sans grand mal, heureusement!

Mais un éclat de rire salua sa chute: Ogotemmé, un jeune Dogon, l'avait vu et se moquait... Le Peuhl, vexé, ramassa une pierre. Le Dogon avait sa lance en main... En temps normal la bataille aurait été inévitable: un Peuhl ne peut accepter d'être tourné en ridicule par un Dogon! Mais était-ce l'influence de la soif, ou peut-être la fraternité des souffrances partagées? Nul d'entre eux n'avait envie de se battre. Ogotemmé, 
après quelques secondes de silence, posa sa lance à terre, et dit: «Tu es fils de la plaine, je suis fils du rocher. Regarde...» Et il se mit à l'escalade. Mamadou lui cria: «Apprends-moi et je t'apprendrai à monter à cheval.» Les yeux d'Ogotemmé brillèrent... «Viens». Et l'un aidant l'autre, ils recommencèrent l'escalade. Peu à peu Mamadou s'enhardissait, avait moins besoin des conseils de son camarade. Ils s'arrêtèrent, une dizaine de mètres au-dessous de la grotte, et, s'asseyant côte à côte sur une aspérité regardèrent la plaine...

Soudain, prêtant l'oreille, Mamadou saisit le bras de son compagnon: «Ecoute!» Ogotemmé se figea: là-haut dans la grotte ils entendaient le murmure de l'eau! «L'eau est là», murmura Ogotommé, «pourquoi ne jaillit-elle pas?» «Allons voir», dit Mamadou. Mais Ogotemmé eut un sursaut de frayeur: «Tu es fou, les Dieux le défendent! Cette grotte est notre mère, si nous entrions là-dedans, ce serait comme si... comme si un Dogon violait sa propre mère, tu comprends?» Et épouvanté à cette idée, Ogotemmé ajouta: «Redescendons!» Il entreprit de descendre, mais, levant bientôt la tête pour aider Mamadou, il vit que celuici, au lieu de descendre, montait, s'approchant de l'orifice béant... Atterré, Ogotemmé hurla: «Redescends!» Mamadou lui fit un léger signe de la main, et entra dans la grotte.... Ogotommé, claquant des dents, se cramponna à son rocher, dans l'attente de quelque cataclysme...

En bas, dans la plaine, des enfants Dogon avaient vu la scène: ils coururent au village porter la nouvelle: Ogotemmé avait aidé un Peuhl à pénétrer la grotte sacrée!

Devant une si terrible profanation, le Grand Hogon réunit le Conseil des Anciens... Comment punir le traître? La mort même serait trop douce...

Pendant ce temps, Ogotommé, tremblant de tous ses membres, descendait lentement de rocher en ro- cher. Au pied de la falaise, quelques Peuhls l'attendaient. «Qu'as-tu fait de Mamadou?» Ogotemmé s'assit, la tête entre les mains... Les Peuhls devinrent menaçants... Soudain, un cri leur fit lever la tête: Mamadou sortait de la grotte, criant joyeusement... Il tordait son pagne trempé et des gouttes d'eau tombaient au vent.... En descendant, il faillit tomber, mais Ogotemmé remonta l'aider, et quelques instants plus tard, tous deux sautaient sains et saufs au milieu du cercle des Peuhls. Exultant, Mamadou ne savait que rire et dire: «L'eau est là!» Enfin, il s'expliqua, pendant qu'Ogotemmé le regardait, les yeux arrondis d'étonnement...

Quelques instants Plus tard, Ogotemmé rentrait au village dogon. Son attitude était bizarre: on le sentait assez inquiet, mais malgré tout, pénétré d'importance. En rien il n’avait l'air d'un coupable! Sur la place centrale sous l'auvent appelé Maison des Anciens, tous les vieux, les sages du village, siégeaient en tribunal. Tout autour, en cercle, les femmes et les jeunes, tous ceux qui n'avaient pas droit au chapitre. Ogotemmé s'avança, entra dans le cercle. Le grand Hogon écumait de rage. Il exposa l'accusation d'impiété, de trahison vis-à-vis de la religion et de tous les ancêtres, dont Ogotemmé s'était rendu coupable. Le plus ancien s'arrête au moment où, dans sa rage, il allait frapper le jeune homme; et il demanda à Ogotemmé d'exposer sa défense. Ogotemmé répondit: «Notre religion nous dit que tout Dogon qui entre dans la grotte des Origines sera puni comme un inceste et foudroyé par les Dieux... Mais... un Dogon est-il entré dans la grotte? Non. Un Peuhl y est entré. Faut-il que je sois puni pour cela? Les Dieux eux-mêmes ont répondu qu'il n'y avait pas sacrilège, puisque le Peuhl est sorti sain et sauf. Non seulement il est sorti de la grotte, mais il a retrouvé l'eau.» 
A ces mots, le cercle des femmes et des jeunes se fit plus serré, plus attentif... Même chez les sages, un mouvement de surprise et d'intérêt troubla leur impassibilité. Le sorcier bondit: «La mort, la mort seule...» Le plus Ancien le coupa: «Le Conseil est là pour décider...» Et Ogotemmé expliqua: «Le plafond de la grotte a été heurté par un tronc d'arbre, venu je ne sais d'où avec le courant d'eau, et s'est effondré, bouchant la grotte. Derrière ce barrage, l'eau a monté, a trouvé une autre faille, et s'en va je ne sais où, dans la roche de la falaise»... Le sorcier, excédé, secoua la tête: «Personne ne te croit!»

Mais Ogotommé, lui, sentait que tout le monde le croyait, attendait la suite, ce qu'il allait dire... «Alors... Alors...» Mais se rendant compte de l'énormité de la chose, il se troubla, s'arrêta... puis, comme quelqu'un qui se jette à l'eau, il enchaîna très vite: "Alors le Peuhl dit, en travaillant avec des dabas, on peut creuser le barrage, le démolir, et l'eau reviendra... comme avant!» Un silence stupéfait accueillit la fin de son discours. Puis, le sorcier hurla: «Tu veux tous nous entraîner dans ton impiété? Tu veux nous amener tous à insulter nos Dieux?» Ogotemmé répondit: «Je veux de l'eau pour tout le village.» Un murmure d'approbation monta du cercle des femmes et des jeunes. Le Doyen apostropha Ogotemmé: «Tu sais bien que nous ne pouvons entrer dans cette grotte, nul d'entre nous ne peut braver cette interdiction qui a été signifiée par les Dieux à nos ancêtres. Un fils ne peut violer sa mère, et nous sommes fils de la falaise.»

«Certes, répondit Ogotommé, qui, sentant la sympathie diffuse de l'assistance, avait retrouvé son aplomb. Nous, Dogons, nous ne pouvons entrer dans la grotte, mais les Peuhls le peuvent sans encourir la colère des Dieux: la preuve, un Peuhl l'a fait et en est sorti. Alors pourquoi nous, qui sommes les fils de la Falaise, les fils du Rocher, n'aiderions-nous pas les
Peuhls en leur disant: nous vous montons jusqu'à la grotte, nous vous prêtons nos instruments, nos dabas, et vous, dans la grotte, vous détruisez le barrage, et l'eau jaillira pour tous!»

Le Grand Sorcier resta sans voix devant une telle proposition: la fureur l'étouffait. Les Sages ne savaient que dire... Alors une femme s'avança, et montrant son sein flétri, son enfant presque mort de soif: « $\mathrm{Si}$ les Dieux ne peuvent nous rendre l'eau», murmurat-elle, «que les hommes nous la rendent!»

Le soir-même, une délégation d'anciens du village dogon s'en alla trouver les chefs peuhls, et, rencontrant Mamadou, Ogotemmé lui fit le clin d'œil qui, dans tous les pays du monde, doit vouloir dire: «Tout va bien!»

DÈs LE LENDEMAIN, LE TRAVAIL commença sur la falaise: les Dogons installaient des cordes, des échelles, les Peuhls grimpaient dans la grotte, armés de dabas... Et l'on descendit les premières outres gonflées d'eau, que se partagèrent les femmes peuhles et les femmes dogonnes.

Le travail fut plus long que Mamadou ne l'avait prévu: le tronc d'arbre, cause de l'effondrement, était resté en travers de la grotte. Il fallait le casser. Mais d'autre part, le plafond de la grotte risquait encore de s'effondrer un jour prochain. Les Peuhls allèrent chercher, au loin dans la plaine, sur les bords de l'ancienne rivière, des troncs d'arbre qu'ils firent tirer par leurs chevaux et avec lesquels ils étayèrent la grotte comme une galerie de mine. Après cela, il suffirait de casser le tronc d'arbre, cause du malheur, en son milieu, et l'eau jaillirait, emportant les pierres du barrage. Mais il fallait que le barrage cède en son milieu: si l'eau jaillissait par un des côtés, elle risquait, prenant l'étayage par derrière, de provoquer un nouvel effondrement. 
Enfin, tout fut prêt; les gros blocs de pierre avaient été enlevés du barrage, le tronc attaqué à la hache en son milieu était devenu assez mince pour être cassé avec l'appoint d'un simple levier. Mais pour manier ce levier? Qui le pousserait serait à coup sûr emporté par le courant! Un système de cordes avait été prévu par les soins conjugués de Mamadou et d'Ogotemmé; et l'on attendit avec confiance le dernier effort. Les Peuhls sortirent de la grotte, avertirent les Dogons qu'il ne restait plus qu'à placer les cordes pour manouvrer le levier, et que cela serait fait le lendemain matin: demain l'eau jaillirait...

Les deux villages dormirent dans l'attente du grand événement... Sommeil agité à coup sûr! Et le sommeil que ne partageaient ni Ogotemmé ni Mamadou... L'amitié, née entre eux dès le premier jour, n’avait fait que croître, et ils étaient devenus inséparables. Pourtant, en cette dernière nuit d'attente, Ogotemmé semblait triste. Ils étaient assis tous les deux près d'un feu au pied de la falaise, et, levant les yeux, Mamadou disait: «Demain l'eau jaillira!» Mais Ogotommé, pensif: «Oui, l'eau jaillira... et dans un an, quand vous reviendrez, vos troupeaux piétineront nos champs, nous taperons sur vos troupeaux, vous défendrez vos bêtes, et nous nous battrons les uns contre les autres...» Mamadou sourit de plus belle: «C’est cela qui te rendait pensif? Ecoute: tu sais que les troupeaux chargent quand ils sentent l'eau, à deux kilomètres de la rivière... et nous ne pouvons alors les arrêter, ils vont droit jusqu'à l'eau. Mais si nous faisions...» Et jusqu'à l'aube, Mamadou et Ogotommé, tête contre tête, examinèrent des dessins que Mamadou traçait sur le sol: un grand trait représentant la rivière, au pied d'une grosse pierre représentant la falaise. De ce trait partait un grand $V$ fait de petites branchettes de bois... Et Ogotommé, dans le jour nouveau, riait en suivant du doigt les branches du V.
MaIS, COMME LES PREMIÈRES lueurs du jour éclairaient la falaise, Mamadou lança un cri d'alarme: du trou de la grotte, l'eau suintait. Si l'eau réussissait à crever le barrage par un côté et non par le milieu, c'était la catastrophe, tout le travail à refaire. "Vite les cordes!» hurla Mamadou... Et portant les câbles tressés, il se rua avec Ogotemmé vers la falaise. Jamais ils ne l'avaient escaladé si vite! Ogotemmé s'arrêta à l'entrée de la grotte, passa les cordes à Mamadou qui entra... et poussa un cri de désappointement: l'eau bouillonnait sur le côté droit, à l'extrémité du barrage... Pas le temps de mettre les cordes, la catastrophe était imminente... «Trop tard», cria Mamadou, «cache toi, Ogotommé, je fais sauter l’arbre!» Et Ogotemmé tremblant d'une religieuse terreur, risqua pour la première fois un œil dans la caverne. Il vit Mamadou, arcbouté poussant de toutes ses forces sur le levier pour casser l'arbre, au centre du barrage... Mais Mamadou seul n'avait pas assez de forces... Il s'acharnait cependant, criant à Ogotemmé: «Pars, cache-toi.» Alors, chez Ogotemmé, l'amitié fut plus forte que la terreur ancestrale: il bondit dans la caverne, vint se mettre au levier auprès de son ami. Un sourire illumina le visage du Peuhl; Ogotemmé y répondit, puis tous deux unirent leurs efforts, et l'arbre cassa. Et l'eau jaillit, en une cascade irisée tombant des 60 mètres de haut, dans la vasque de pierre... Et mêlés aux pierres et aux débris de bois, tombèrent aussi les corps d’Ogotemmé et de Mamadou... de la vasque de pierre à nouveau emplie d'eau, on sortit les corps des deux amis. Mamadou était mort. Ogotemmé respirait encore. On les étendit tous les deux près des braises de leur feu nocturne, et Ogotemmé, aux Peuhls et aux Dogons qui les entouraient, silencieux, il montra du doigt, sur le sable, le dessin qu'avait fait Mamadou dans la nuit, la palissade en $\mathrm{V}$ allant vers la rivière, et il murmura: «Il faut construire ça, tous ensemble... Les 
Peuhls iront chercher du bois... Ils amèneront les troupeaux chaque année devant la bouche ouverte des palissades... Les bêtes n'iront pas dans les champs... Il faut construire... ensemble...»

Peuhls et Dogons jurèrent de construire ensemble, et Ogotemmé mourut auprès de Mamadou.

IL Y A MAINTENANT, s'en allant vers la rivière un grand $\mathrm{V}$ de palissade, et chaque année, c'est dans ce $\mathrm{V}$ que s'engouffrèrent les troupeaux des Peuhls; il y a der- rière les palissades, les champs intacts des Dogons; il y a, jaillissant de la grotte, l'arc irisé de l'eau vivifiante; il y a, au pied de la falaise, une pierre plate marquée d'un croissant, sous laquelle repose le corps de Mamadou, et au cour de la falaise, dans le roc où reposent ses ancêtres, le corps d'Ogotemmé; et la paix est si profonde que Peuhls et Dogons appellent cette vasque où tombe l'eau «le bain des amis» et leurs enfants s'y baignent ensemble, dans les rires et les cris de joie. 